\title{
The role of manual aspiration thrombectomy in the management of STEMI: a TOTALly different TASTE of TAPAS
}

\author{
Goran Stankovic ${ }^{1,2}$, Dejan Milasinovic ${ }^{1}$ \\ ${ }^{1}$ Department of Cardiology, Clinical Center of Serbia, Belgrade, Serbia \\ ${ }^{2}$ Faculty of Medicine, University of Belgrade, Belgrade, Serbia
}

Adv Interv Cardiol 2016; 12, 1 (43): 3-5 DOI: $10.5114 /$ pwki.2016.56942

In the latest ACC/AHA/SCAl guideline update on primary percutaneous coronary intervention $(\mathrm{PCl})$ for patients with ST-elevation myocardial infarction (STEMI), routine use of manual thrombectomy as an adjunct to primary $\mathrm{PCl}$ is not recommended (class III: no benefit, level of evidence A) [1]. The guidelines also state that the usefulness of selective and bailout aspiration thrombectomy in patients undergoing primary $\mathrm{PCl}$ is not well established (class IIb, level of evidence C). A change in the recommendation occurred following publication of two large randomized studies, the TOTAL trial (a trial of routine aspiration ThrOmbecTomy with $\mathrm{PCl}$ versus $\mathrm{PCl}$ ALone in Patients with STEMI) $(n=10$ 732) [2] and the TASTE study (Thrombus Aspiration in ST-Elevation Myocardial Infarction in Scandinavia) $(n=7244)$ [3], which both showed no difference in clinical outcomes between $\mathrm{PCl}$ plus thrombectomy versus $\mathrm{PCl}$ alone. Previously, the smaller $(n=1071)$ randomized TAPAS study (Thrombus Aspiration during Percutaneous Coronary Intervention in Acute Myocardial Infarction Study) [4] had shown that manual thrombectomy was associated with better post$\mathrm{PCI}$ myocardial perfusion, as measured by the myocardial blush grade (MBG), and a reduction in cardiac mortality, although this study was not powered to detect differences in clinical events. However, we believe that there are important observations that should be taken into account when weighing the impact of these trials on everyday practice.

In this issue of Advances in Interventional Cardiology, 2 case reports describe successful application of manual thrombectomy beyond the scope of routine STEMI management (a patient with breast cancer and paraneoplastic syndrome, who suffered non-ST-segment myocardial infarction and a patient with bacterial endocarditis of the aortic valve and anterior STEMI caused by embolization of thrombotic material into the left anterior descending (LAD) coronary artery) [5, 6].

Taken together with the recently published data on the lack of clinical benefit of routine manual thrombectomy, the two described reports seem to raise the following question: Has the role of thrombectomy shifted from being considered part of the routine practice of STEMI treatment to being a non-standard therapeutic tool for an exceptional acute MI patient? To attempt an answer to this question, we analyze the results of the main and secondary publications from the TOTAL and the TASTE trials and relate these findings to the knowledge base from earlier clinical studies that seemed to be in favor of the routine use of manual thrombectomy.

In the TOTAL trial, patients undergoing manual aspiration thrombectomy as an adjunct to primary $\mathrm{PCl}$ had a similar rate of the combined primary endpoint of cardiovascular death, recurrent $\mathrm{MI}$, cardiogenic shock or NYHA IV heart failure at 180 days, as compared with PCI alone $(6.9 \%$ vs. $7.0 \%$, respectively; $\mathrm{HR}=0.99,95 \% \mathrm{Cl}$ : $0.85-1.15, p=0.86$ ) [2]. There were no significant differences in individual components of the primary endpoint or in the rate of stent thrombosis (1.5\% vs. $1.7 \%, p=$ 0.42 ) or target vessel revascularization ( $4.5 \%$ vs. $4.3 \%$, $p=0.77$ ), at 180 days [2]. The crossover rate was $4.6 \%$ from thrombectomy to $\mathrm{PCl}$ alone and $1.4 \%$ from $\mathrm{PCl}$ alone to thrombectomy, while the rate of bailout thrombectomy in the $\mathrm{PCl}$-alone group was 7.1\% [2]. Nevertheless, the on-treatment analysis that compared patients who received thrombectomy irrespective of randomization (both upfront and bailout) with $\mathrm{PCl}$ alone showed no significant difference in the rate of the primary endpoint. At 1 year, the occurrence of the primary composite end-

\section{Corresponding author:}

Goran Stankovic MD, PhD, FACC, FESC, Department of Cardiology, Clinical Center of Serbia, Visegradska 26, 11000 Belgrade, Serbia, phone: +381-3613653, e-mail: gorastan@sbb.rs

Received: 29.12.2015, accepted: 29.12.2015. 
point was the same in patients with thrombectomy vs. $\mathrm{PCl}$ alone (8\% in both groups) [7]. On top of demonstrating neither early nor 1-year benefit of thrombectomy, the TOTAL trial showed an increased incidence of stroke in patients subjected to manual thrombus aspiration (at 30 days: $0.7 \%$ vs. $0.3 \%, p=0.015$; at 180 days: $1.0 \%$ vs. $0.5 \%, p=0.003$; at 1 year: $1.2 \%$ vs. $0.7 \%, p=0.015)[2$, $7,8]$. The greatest difference in the occurrence of stroke was documented in the first $48 \mathrm{~h}$ after $\mathrm{PCl}(0.3 \%$ vs. $0.1 \%, p=0.025$ ) [8]. Thrombus dislodgment from coronary into systemic vasculature may have accounted for early ischemic strokes in patients undergoing thrombectomy. However, the following two findings remain largely unexplained and may be attributed to the play of chance: firstly, the more frequent occurrence of stroke in the period between 90 and 180 days after $\mathrm{PCl}$; secondly, the excess of hemorrhagic stroke in the thrombectomy group, which may have been the consequence of the very low overall number of events $(n=12)$ and/or difficulties in differentiating between primary hemorrhagic and ischemic strokes with hemorrhagic transformation.

Adding to the evidence from the TOTAL trial, the lack of clinical benefit of manual aspiration thrombectomy was confirmed by the TASTE study, which like the TOTAL trial was powered to assess the impact of thrombectomy on clinical events. In the TASTE study, 11709 patients were screened but only 7244 (60\%) were randomized. Importantly, the remaining 4580 patients were followed in a parallel registry and $24.8 \%$ of them underwent thrombectomy. The primary endpoint of all-cause mortality at 30 days did not differ between patients assigned to thrombectomy vs. $\mathrm{PCl}$ alone $(2.8 \%$ vs. $3.0 \%$ in randomized patients, while it was $10.9 \%$ vs. $10.5 \%$ in two registry arms) [3]. Other clinical outcomes, including reinfarction, stent thrombosis and target vessel/lesion revascularization, were also similar between the treatment groups. At 1 year, there was also no difference in mortality $(5.3 \%$ vs. $5.6 \%$, in the thrombectomy vs. $\mathrm{PCl}$ alone patients respectively) or other clinical outcomes [9]. The TOTAL and the TASTE trials had concordant results, although there were design differences (for example, randomization af- ter angiography in the TASTE trial and before angiography in the TOTAL trial, and the time from symptom onset to randomization, which was $<24 \mathrm{~h}$ in the TASTE trial and $<12 \mathrm{~h}$ in the TOTAL trial).

The absence of thrombectomy-associated improvement in clinical outcomes in the TOTAL and the TASTE trials contradicted previously published findings of the TAPAS study, which had suggested reduced 1-year cardiac mortality in patients treated with thrombus aspiration compared to conventional $\mathrm{PCl}(3.6 \%$ vs. $6.7 \%$, respectively, $p=0.02$ ) [10]. Although the results of the TAPAS and the TOTAL trials seem to be conflicting, a closer look might reveal a common set of evidence on the impact of aspiration thrombectomy in patients with STEMI. Namely, in both trials thrombectomy resulted in an improvement of parameters associated with better myocardial perfusion (complete ST-segment resolution (STR) in both studies, MBG $\geq 2$ in TAPAS and less distal embolization in TOTAL). Hence, the apparent discrepancy between the studies appears to originate mainly from the fact that TAPAS was not powered to detect differences in clinical outcomes.

It is difficult to explain why manual thrombectomy did not have an impact on the outcome of patients undergoing primary PCI for STEMI. The technique of aspiration thrombectomy is based on the mechanical removal of coronary thrombi prior to stent implantation, and was believed to confer patient benefit in two distinct aspects: firstly, via improvement in myocardial perfusion, which could consequently lead to infarct size reduction and better clinical outcomes; and secondly, by ameliorating thrombus burden prior to stent implantation, thus decreasing the rate of stent-related complications (i.e. due to underexpansion or malapposition). Surrogates of myocardial reperfusion, such as complete STR, have consistently been improved by thrombectomy across the trials, even including the overall negative TOTAL trial. However, thrombectomy was not associated with reduced infarct size, as assessed by magnetic resonance imaging [11] or SPECT [12]. Similarly, the theoretical potential to reduce thrombus burden at the site of stent implantation was

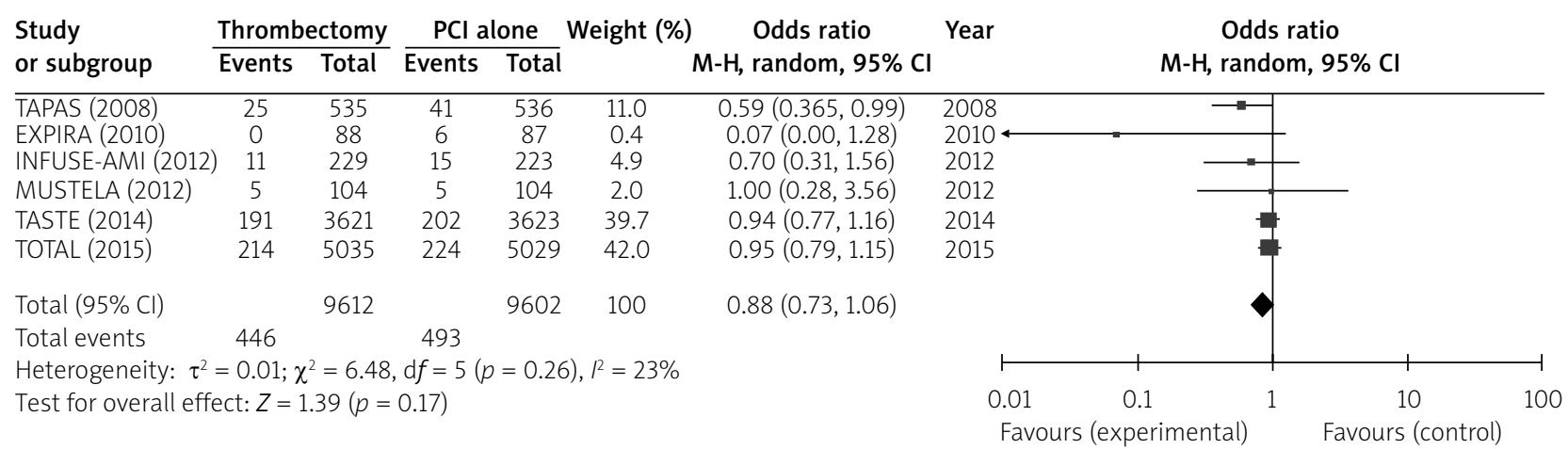

Figure 1. Forest plot showing no significant difference in all-cause mortality at least 12 months after $\mathrm{PCl}$, in STEMI patients with vs. without manual aspiration thrombectomy as adjunct to primary $\mathrm{PCI}$ 
not fulfilled, as evidenced by the TOTAL-OCT substudy, which showed no difference in thrombus burden before stenting [13]. To reveal potential thrombectomy benefits at a longer-term follow-up, we performed a meta-analysis of randomized trials that reported at least 12 months all-cause mortality $[7,9,11,14,15]$, which confirmed the absence of a positive effect at this time interval ( $O R=$ $0.88,95 \% \mathrm{Cl}: 0.73-1.06, p=0.17$ ) (Figure 1).

In conclusion, manual aspiration thrombectomy has been associated with improved myocardial perfusion, most notably by reducing the rate of MBG 0 or 1 in the TAPAS trial, albeit without translation into clinical benefit, as shown in the TASTE and TOTAL trials, and supported by a recent meta-analysis [16]. The explanation for this may be found in the generally highly complex relationship between surrogates and hard clinical endpoints [17]. In the particular context of a STEMI patient, thrombectomy-associated improvement in surrogates of myocardial perfusion, such as STR or MBG, is only one piece of a complex puzzle that also includes microvasculature-mediated flow resistance, cardiomyocyte response to ischemia and reperfusion injury, and stent/ procedure-related complications, such as bleeding and contrast-induced acute kidney injury.

Despite the lack of clinical benefit associated with its routine upfront use, manual aspiration thrombectomy appears to remain a useful tool in the interventional cardiologist's armamentarium. However, we expect that the rate of manual thrombectomy during primary $\mathrm{PCI}$ will decrease to $10 \%$ to $25 \%$ of cases (between the crossover rate in the TOTAL trial and the rate of use in the registry patients in the TASTE trial) and will be reserved for carefully selected cases with an anticipated high risk of distal embolization or with no-reflow after balloon angioplasty or stent implantation.

\section{Conflict of interest}

The authors declare no conflict of interest.

\section{References}

1. Levine GN, O'Gara PT, Bates ER, et al. 2015 ACC/AHA/SCAI Focused Update on Primary Percutaneous Coronary Intervention for Patients With ST-Elevation Myocardial Infarction: An Update of the 2011 ACCF/AHA/SCAI Guideline for Percutaneous Coronary Intervention and the 2013 ACCF/AHA Guideline for the Management of ST-Elevation Myocardial Infarction: A Report of the American College of Cardiology/American Heart Association Task Force on Clinical Practice Guidelines and the Society for Cardiovascular Angiography and Interventions. J Am Coll Cardiol 2015 Oct 21 [Epub ahead of print]; doi: 10.1016/j. jacc.2015.10.005.

2. Jolly SS, Cairns JA, Yusuf S, et al. Randomized trial of primary PCI with or without routine manual thrombectomy. N Engl J Med 2015; 372: 1389-98

3. Frobert O, Lagerqvist B, Olivecrona GK, et al. Thrombus aspiration during ST-segment elevation myocardial infarction. N Engl J Med 2013; 369: 1587-97.
4. Svilaas T, Vlaar PJ, van der Horst IC, et al. Thrombus aspiration during primary percutaneous coronary intervention. $N$ Engl J Med 2008; 358: 557-67.

5. Ciećwierz D, Mielczarek $M$, Jaguszewski $M$, et al. The first reported aspiration thrombectomy with a guide extension mother-and-child catheter in STEMI due to bacterial vegetation coronary artery embolism. Postep Kardiol Inter 2016; 12: 71-3.

6. Dąbrowski M, Tyczyński P, Bęćkowski $M$, et al. Is there still a place for thrombectomy? Postep Kardiol Inter 2016; 1269-70.

7. Jolly SS, Cairns JA, Yusuf S, et al. Outcomes after thrombus aspiration for ST elevation myocardial infarction: 1-year follow-up of the prospective randomised TOTAL trial. Lancet 2015 Oct 12 [Epub ahead of print]; doi: 10.1016/S0140-6736(15)00448-1.

8. Jolly SS, Cairns JA, Yusuf S, et al. Stroke in the TOTAL trial: a randomized trial of routine thrombectomy vs. percutaneous coronary intervention alone in ST elevation myocardial infarction. Eur Heart J 2015; 36: 2364-72.

9. Lagerqvist B, Frobert O, Olivecrona GK, et al. Outcomes 1 year after thrombus aspiration for myocardial infarction. N Engl J Med 2014; 371: 1111-20.

10. Vlaar PJ, Svilaas T, van der Horst IC, et al. Cardiac death and reinfarction after 1 year in the Thrombus Aspiration during Percutaneous coronary intervention in Acute myocardial infarction Study (TAPAS): a 1-year follow-up study. Lancet 2008; 371: 1915-20.

11. Stone GW, Witzenbichler B, Godlewski J, et al. Intralesional abciximab and thrombus aspiration in patients with large anterior myocardial infarction: one-year results from the INFUSE-AMI trial. Circ Cardiovasc Interv 2013; 6: 527-34.

12. Kaltoft A, Bottcher M, Nielsen SS, et al. Routine thrombectomy in percutaneous coronary intervention for acute ST-segment-elevation myocardial infarction: a randomized, controlled trial. Circulation 2006; 114: 40-7.

13. Bhindi R, Kajander OA, Jolly SS, et al. Culprit lesion thrombus burden after manual thrombectomy or percutaneous coronary intervention-alone in ST-segment elevation myocardial infarction: the optical coherence tomography sub-study of the TOTAL (ThrOmbecTomy versus PCI ALone) trial. Eur Heart J 2015; 36: 1892-900.

14. De Carlo M, Aquaro GD, Palmieri C, et al. A prospective randomized trial of thrombectomy versus no thrombectomy in patients with ST-segment elevation myocardial infarction and thrombus-rich lesions: MUSTELA (MUltidevice Thrombectomy in Acute ST-Segment ELevation Acute Myocardial Infarction) trial. JACC Cardiovasc Interv 2012; 5: 1223-30.

15. Sardella G, Mancone M, Canali E, et al. Impact of thrombectomy with EXPort Catheter in Infarct-Related Artery during Primary Percutaneous Coronary Intervention (EXPIRA Trial) on cardiac death. Am J Cardiol 2010; 106: 624-9.

16. Elgendy IY, Huo T, Bhatt DL, Bavry AA. Is aspiration thrombectomy beneficial in patients undergoing primary percutaneous coronary intervention? Meta-analysis of randomized trials. Circ Cardiovasc Interv 2015; 8: e002258.

17. Weintraub WS, Luscher TF, Pocock S. The perils of surrogate endpoints. Eur Heart J 2015; 36: 2212-8. 